\title{
Aprendizagem Baseada em Projetos: o caso Health Simulator
}

Recibido: 1 Julio 2015 - Revisado: 30 Septiembre 2015

Aceptado: 30 Octubre 2015 - Publicado: 30 Diciembre 2015

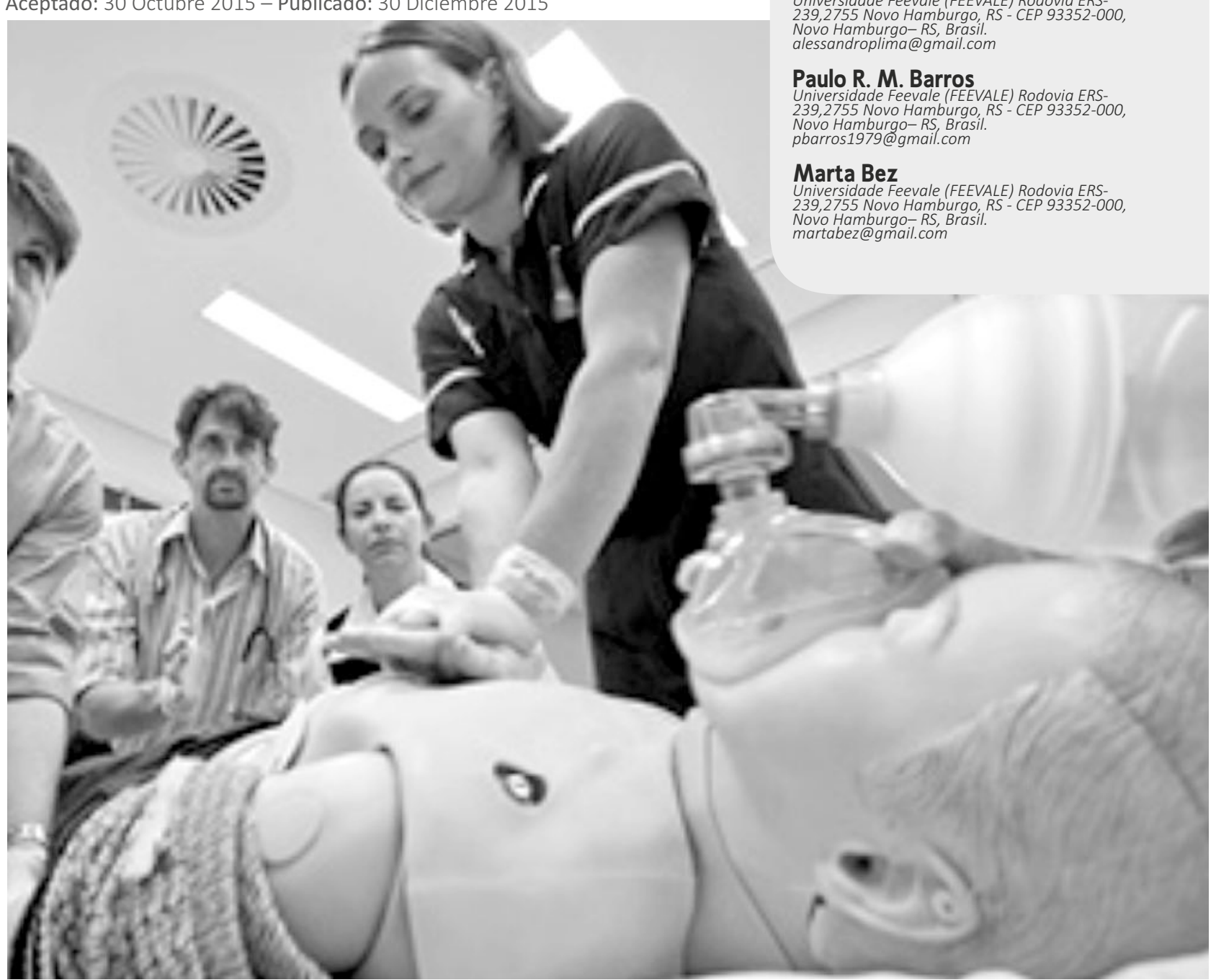

Resumo: Este artigo apresenta um exemplo de uso da Aprendizagem Baseada em Projetos (ABP) para o desenvolvimento de um Simulador do tipo Paciente Virtual denominado Health Simulator. Apresenta-se a teoria da ABP que embasou a estruturação de um trabalho extraclasse que vem sendo realizado ao longo dos últimos dois anos, bem como o produto gerado e os resultados alcançados até o momento.

Abstract: This article presents an example of using Project Based Learning (PBL) for the development of a kind of Virtual Patient Simulator- Health Simulator. It presents the theory of PBL that based structuring an extra-class work that has been done over the past two years, as well as the product generated and the results achieved to date. 


\section{INTRODUÇÃO}

A Aprendizagem Baseada em Projetos (ABP) é uma metodologia de ensino e aprendizagem onde o aluno deve ser o agente na produção do seu conhecimento. Ela pode ocorrer tanto em aulas formais, onde professores organizam assuntos e conteúdos que serão desenvolvidos em aula, normalmente com trabalhos em grupo, quanto fora da sala de aula. Desta forma, rompe-se a forma tradicional e rígida já desenvolvida dos conteúdos, sendo os mesmos incorporados durante o desenvolvimento do projeto.

O filósofo americano John Dewey (1859-1952) foi um dos precursores da aprendizagem baseada em projetos, que procurava demonstrar o "aprender mediante o fazer" (MASSON, MIRANDA, MUNHOZ JR E CASTANHEIRA, 2012). Esta metodologia é considerada construtivista, pois permite aos alunos adquirirem conhecimentos, habilidades e valores através de um processo gradativo de investigação, "estruturado em torno de questões complexas e autênticas, aplicadas na vida real, com produtos e tarefas cuidadosamente planejados" (BIE, 2008). Além disso, o escopo desse método respalda na diversidade informacional, ao invés da unicidade de fonte de conhecimento que se resvale unicamente na figura do professor (PAPINCZACK, 2009).

Torna-se, nesta metodologia, importante o desenvolvimento de estratégias onde metas e padrões curriculares específicos sejam incorporados, contribuindo, desta forma, no desenvolvimento das habilidades e de hábitos de aprendizagem contínua. A análise dos projetos a ser implementada deve ser feita considerando que a educação, ao longo de toda a vida, deve basear-se em quatro pilares: aprender a conhecer, aprender a fazer, aprender conviver e aprender a ser (DELORS, 2006). A experiência prévia e o envolvimento ativo dos alunos são fundamentais para a motivação e aquisição de conhecimentos. Assim, conscientizando os alunos do que eles sabem e do que necessitam aprender, é possível motiva-los na busca de informações que complementem seu conhecimento. Conforme Markham et al. (2008), a aprendizagem é uma atividade social, que ocorre em diferentes contextos, como de cultura, comunidade ou de experiências já vivenciadas, portanto, pode ser considerada uma atividade social.

Existem diversos casos de sucesso de uso dessa abordagem, como em Mafra (2014) que utilizou no curso de Engenharia para a produção de manuais uso de jogos educacionais, tecnologias móveis e comunidade virtual com sujeitos em tratamento oncológico técnicos a partir da interação entre pares. Segundo o autor, a condição ideal para a aprendizagem foi aquela em que os alunos sentiram o senso de competência, domínio e autonomia. Nobre et al. (2006) desenvolveram um projeto para o ensino de software embarcado para um veículo aéreo não tripulado à uma turma de Engenharia Eletrônica. Os autores destacam o fato dos alunos poderem experimentar situações concretas e práticas que encontrarão na vida profissional como engenheiros da computação, mediante equipes distribuídas atuando simultaneamente em um único projeto.

Este artigo apresenta um exemplo de uso da Aprendizagem Baseada em Projetos, realizada de forma extraclasse, com alunos dos cursos de design, jogos digitais e computação. O projeto apresentado aqui, consta do desenvolvimento de um simulador do tipo Paciente Virtual (ORTON; MULHAUSEN, 2008) denominado de Health Simulator (LIMA AYMONE, 2015) cujo objetivo é desenvolver o raciocínio clínico e diagnóstico do aluno da área da saúde, acompanhando sua conduta e evolução durante a simulação.

O grupo é composto por três professores, alunos e egressos voluntários. Coube aos professores a estruturação e organização do projeto, distribuição das tarefas e acompanhamento das atividades, auxiliando no caso de dúvidas e instigando os participantes na busca de material tanto bibliográfico, 
quanto por projetos similares. A experiência no desenvolvimento de outros simuladores deste tipo (SEBASTIANI ET AL., 2014), (SEBASTIANI ET AL., 2012), (MARONI ET AL., 2013), (BARROS ET AL., 2012), (FLORES ET AL., 2012) por parte de alguns membros da equipe, facilitou a condução dos trabalhos e percepção dos problemas que poderiam ocorrer no projeto atual.

Na seção 2 deste artigo é apresentada a base teórica para o desenvolvimento de simuladores do tipo Paciente Virtual. A seção 3 apresenta a metodologia utilizada. O desenvolvimento é descrito na seção 4, seguido das considerações finais.

\section{SIMULADORES DO TIPO PACIENTE VIRTUAL}

A falta de preparo dos profissionais da área da saúde, segundo Bradley (2006), demonstra as falhas nos métodos de aprendizagem de jovens médicos. As restrições de tempo de trabalho têm levantado preocupações sobre o treinamento médico e a quantidade de experiência clínica a que o estudante é exposto, podendo, a simulação apoiar o processo de ensino e aprendizagem.

Simuladores para o ensino médico são ferramentas que possibilitam que os educadores tenham total controle em cenários clínicos pré-selecionados, descartando, nessa fase de aprendizagem, os riscos potenciais ao paciente (ZIV ET AL., 2005). Para que a simulação seja fiel a realidade, segundo Bradley (2006), aspectos como as expectativas da sociedade, as responsabilidades políticas e a regulamentação da profissão devem ser levadas em consideração.

Foi realizada uma revisão sistemática sobre artigos que abordassem como tema "Simuladores". Além disso, a equipe de projeto conta com a experiência em dois projetos anteriores na mesma linha de trabalho: 0 SimDeCS e o SIACC.
O Simulador Inteligente para a tomada de Decisões em Casos de Saúde- SimDeCS, é um projeto que foi desenvolvido no período de 2011 a 2013 em uma parceria entre duas instituições de ensino superior. Este é um simulador do tipo Paciente Virtual no formato de um jogo sério. Formalismos como Redes Bayesianas (RB) (PERL, 1998), Diagrama de Influência (DI) (ZENG; XIANG, 2010), (SUN ET AL., 2010), Sistemas Multiagentes (SMA) e Estratégia Pedagógica (EP) (FLORES ET AL, 2005) conferem ao SimDeCS robustez e uma estrutura científica que permitiu que diversas investigações fossem realizadas. A arquitetura foi desenvolvida com quatro fases distintas: a) o conhecimento é estruturado no formato de Redes Bayesianas pelo especialista; b) os casos clínicos são desenvolvidos pelos professores com base nas redes bayesianas; c) a interface do aluno que aparece no formato de um jogo sério; e o feedback fornecido ao aluno a cada iteração. Através do SimDeCS alunos de medicina tem a possibilidade de análise de vários casos clínicos, apoiando sua aprendizagem, facilitando o desenvolvimento de suas habilidades técnicas, raciocínio diagnóstico e condutas formuladas. Atualmente, existe uma grande quantidade de material publicado que apresenta este simulador, tanto sobre o desenvolvimento do projeto, quanto a forma de desenvolvimento dos casos clínicos pelos professores, resolução por parte dos alunos e validação com professores e alunos. (BARROS ET AL., 2011), (FLORES ET AL., 2012), (BARROS ET AL., 2012), (BEZ, 2013).

O Sistema Interdisciplinar de Análise de Casos ClínicosSIACC é um tipo de Paciente Virtual que está sendo utilizado em uma Instituição de Ensino superior na área da saúde desde 2009. Este é um sistema web utilizado nas aulas do curso de medicina. Os casos desenvolvidos no SIACC são organizados com base nas seguintes informações: história, exame físico, dados de laboratório e imagens. O aprendizado das principais patologias vivenciadas da atuação profissional médica está disponível no formato de conteúdo e de perguntas que ocorrem no decorrer do 
caso, acompanhado de links para textos extras e informações adicionais. O sistema e validação do mesmo estão descritos em Sebastiani, Flores e Bez (2010), Flores, Bez e Bruno (2011), Sebastiani et al. (2012).

O levantamento bibliográfico proveniente da revisão sistemática, mais a experiência da equipe no desenvolvimento dos simuladores SIACC e SimDeCS, permitiu que se estruturasse o novo simulador. A metodologia e o simulador em desenvolvimento (Health) são apresentados na sequência.

\section{METODOLOGIA UTILIZADA NO PROJETO}

Diversas áreas do conhecimento estão envolvidas no desenvolvimento do Health Simulator, categorizandoo como um projeto interdisciplinar. Para um projeto desta natureza, o processo de Aprendizagem Baseada em Projetos ( $A B P$ ) parece ser aquela que melhor se aplica, tomando a metodologia Projeto E (PROJETOE, 2015) como base, elaborada por Meurer. Esta metodologia busca oferecer um ensino de aprendizagem amigável e engajado por parte dos alunos. Neste sentido, toda a produção do projeto Health busca motivar seus integrantes, oferecendo condições de envolvimento, investigação, colaboração, discussão e reflexões acerca da produção que, ao fim, oportuniza-se ações de planejamento projetuais.

Sendo assim, as etapas da metodologia de Projeto $E$ como método de APB, define-se por: Contextualização, - momento em que se gera um contexto cerca do projeto; Desconstrução entendimento estrutural de projetos similares; Verificação- lista de requisitos necessários a execução do projeto; Reconstrução - definição de ferramentas, cenários, wireframes e fluxos; Identidade- definição e identidade estética do projeto; Diferenciação - testes de diferenciação com o projeto; Desenvolvimento regras de negócio e modelo funcional navegável; Validação- testes com usuários.
Toda a produção prática das etapas de desenvolvimento deste projeto está ancorada no método proposto por Novak (2010) sob a metodologia de Projeto E. Na fase de conceito, por exemplo, definem-se os conceitos acerca do jogo, desde os cenários, personagens, interfaces de hud ou mesmo a animação dos personagens. Na definição de conceito para o projeto Health, optou-se por utilizar um conjunto extenso de mood boards para representar a construção de personagens e cenários (LIMA, AYMONE, 2015).

O gerenciamento da produção em massa de assets customizáveis para o Health é realizada utilizando-se de métodos ágeis (LIKER, 2005), que são definidos como interativos e incrementais, adotando, em cada fase evolutiva do projeto, um momento para revisões e ajustes antes de prosseguir aos demais estágios (TELES, 2006). Dentro dos métodos ágeis, segundo Lacerda et al. (2004), existe o enfoque na adaptação, onde as atividades são administradas conforme a demanda. Scott et al. (2014) defendem que ao utilizar métodos ágeis são percebidas características individuais que afetam o resultado de produção das equipes.

O grupo de desenvolvimento do Health Simulator se reúne uma vez por semana para apresentar resultados, questionar, melhorar e adaptar as rotinas desenvolvidas.

\section{DESENVOLVIMENTO DO HEALTH SIMULATOR}

O Health Simulator é composto por duas partes distintas e complementares: o sistema de geração da informação e o jogo sério. O grupo foi dividido em duas equipes, cada uma responsável por uma destas partes, mas estando em constante contato, analisando o cronograma de atividades e apresentando estudos realizados em cada etapa. As duas partes são identificadas e explicadas na sequência. 


\subsection{O jogo sério}

Esta parte do simulador é destinada aos alunos. Possui cenários relacionados a espaços de atendimento a saúde e personagens que possam representar tanto profissionais da área quanto pacientes.

No projeto Health Simulator está prevista a construção volumosa de elementos de arte ou assets, por esta razão, utiliza-se a filosofia e princípios ágeis de desenvolvimento de software para melhor conduzir os trabalhos. Os valores de Lean (WORMACK, 2004) são interpretados para o desenvolvimento de modelos tridimensionais e digitais. Existem quatro categorias de personagens: Médicos, Pacientes, Enfermeiros e Dentistas, todos nos dois gêneros: masculino e feminino. Os personagens podem ser das etnias, branca, negra, índia e asiática, com idades variadas (bebês, crianças, adolescentes, adultos ou idosos). Para os personagens de atendimento, serão só adultos. Existe também para cada personagem a possibilidade deste ser magro, normal ou obeso.

Um estudo aprofundado sobre as características dos profissionais da saúde foi realizado para o desenvolvimento dos modelos tridimensionais que devem compor o Health Simulator. Com isso, tem-se, aproximadamente, 300 modelos de personagens apenas para a categoria de pacientes a serem desenvolvidos, motivo pela qual se optou por uma metodologia de desenvolvimento que agilizasse sua criação, minimizando o tempo para que fossem incorporados à engine do jogo.

Para os cenários utilizou-se um sistema de reaproveitamento de modelos, sendo esses consultórios ou hospitais das classes A, B ou C e um hospital do Sistema Único de Saúde (SUS). A partir de pranchas de mood board e planilhas com os principais itens dos cenários, o tipo de cenário a ser desenvolvido, o estilo visual e outros dados técnicos, foi possível facilitar a sua produção. Os modelos são compartilhados, permitindo a criação de novos cenários, com poucos ajustes. O desenvolvimento foi iniciado pela modelagem dos itens em 3D com o software 3D Studio Max.

O processo de desenvolvimento iniciou com os objetos que os cenários exigem (maca, mesa de atendimento, cadeiras, equipamentos, entre outros), modelando a malha e, em seguida, realizando o mapeamento das texturas. Um exemplo de mood board e de profissional da saúde são apresentados na Figura 1. Na Figura 2, um exemplo de mood board de cenário e o mesmo modelado são demonstrados.

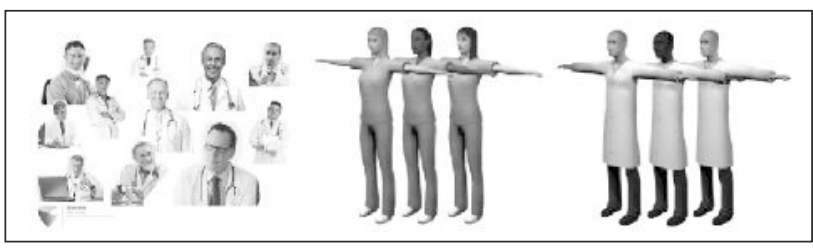

Figura 1. Mood bord de profissional da saúde e modelos 3D projetados.

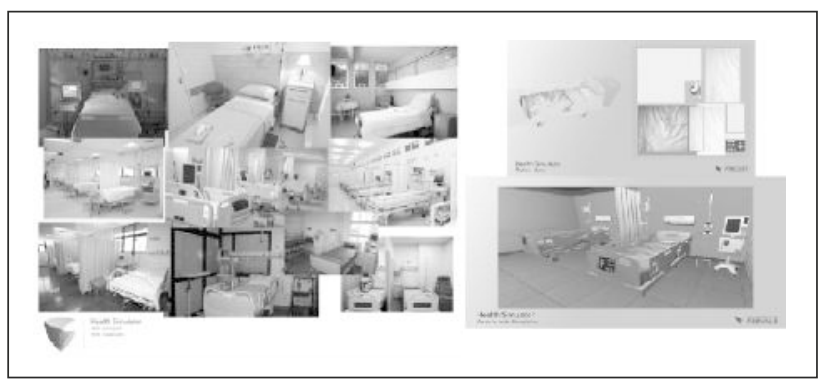

Figura 2. Mood board e cenário desenvolvido.

A engine Unity é a ferramenta utilizada para o desenvolvimento da versão do jogo sério. Os personagens e os cenários estão sendo incorporados neste ambiente e animados. Quanto as interfaces do jogo, contendo menus e hud de respostas para o usuário, levou-se em consideração os preceitos da metodologia de Projeto E (MEURER; SZABLUK, 2010), a qual derivam de Garret (2003) e Preece et al. (2005).

\subsection{Sistema de geração da informação}

O sistema de geração da informação é dividido em três etapas distintas: Modelagem do conhecimento; 
Interface de administração e Serviço Web de comunicação.

Na modelagem do conhecimento, é delimitado o conhecimento a ser representado pelo especialista, com base em diretrizes clínicas (orientação e delimitação do conteúdo a ser desenvolvido a partir de evidências). Um modelo estatístico de representação do conhecimento é construído através de uma rede bayesiana, uma das abordagens reconhecidas para tomada de decisão em medicina (HIGGS ET AL, 2008). Essas são utilizadas como base de conhecimento, para que, posteriormente, os professores possam realizar a montagem dos casos clínicos, elencando as possíveis variáveis, como diagnósticos, sintomas e as suas probabilidades conforme a relação entre elas.

A Interface de administração é a parte do sistema onde o professor tem acesso a variáveis como sinais e sintomas presentes na rede, possíveis históricos anteriores do paciente, bem como, exames físicos e complementares. Conforme a seleção destas variáveis, o sistema deve apresentar o desfecho do caso clínico, com os diagnósticos e condutas, conforme as probabilidades propagadas na rede. Isso facilita o processo e a elaboração de cada caso clínico. Os casos clínicos elaborados são armazenados no Banco de Dados (BD) juntamente com informações pertinentes a modelagem do caso.

Conforme apresentado na Figura 3, por padrão, existe em cada tela um cabeçalho com um menu de acesso à informações para administradores e professores (LIMA, AYMONE, 2015). A direita, há opções de login/logout no sistema e o botão Play, que possibilita ao professor executar o caso no formato do jogo. No centro da tela são apresentados os campos a serem preenchidos, e abaixo, a migalha, que permite a localização do usuário no sistema. A navegação entre as telas é realizada através do menu ou das opções Etapa Anterior e Próxima Etapa. As principais funções do sistema são identificados por ícones de Edição, Gravação e Exclusão.

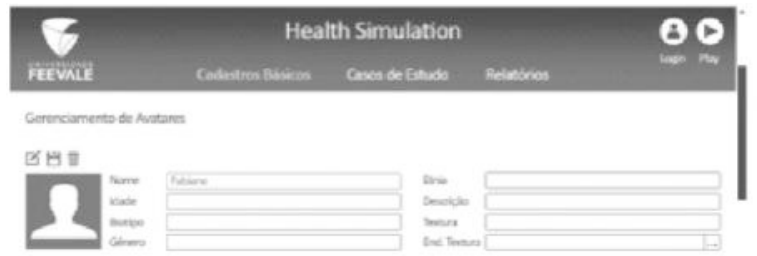

Figura 3. Recorte da tela de cadastro de Avatares.

O Serviço Web de comunicação lê os casos no Banco de Dados e os apresenta aos alunos no formato de jogo. Os casos clínicos estão sendo desenvolvido com base em uma das soluções mais utilizadas para integração de sistemas e comunicação entre aplicações diferentes, permitindo a maior compatibilidade de softwares desenvolvidos em diferentes plataformas (SOMMERVILLE, 2011). Os Serviços Web fornecem uma solução que define um padrão para a troca de mensagens entre as aplicações cliente (jogo) e um fornecedor do serviço (sistema de geração da informação).

Utilizou-se a arquitetura RESTful/MVC4 (MICROSOFT, 2014) para o desenvolvimento do Serviço Web do simulador. Esta é uma arquitetura de transferência de objetos representacionais que fornece serviços de acesso a recursos hospedados em um servidor. As informações da aplicação e de transações foram estruturadas no formato JSON (JavaScript Object Notation) (JSON, 2014). Esta contém um pequeno conjunto de operações para que informações dos recursos sejam manipuladas.

\subsection{Objetivos atingidos}

O projeto foi iniciado com uma equipe pequena composta por um professor e dois egressos do curso de Computação. Logo foi disseminado na instituição, recebendo aderência da comunidade acadêmica, principalmente do curso de jogos digitais e computação. A divulgação do mesmo ocorre, desde seu início, através de palestras internas, denominadas Semana Acadêmica e Hora da Tecnologia. 
Atualmente, o grupo conta com mais de 15 participantes, sendo três professores, egressos e alunos de diversos cursos, que no decorrer do projeto foram se incorporando a equipe. Os conhecimentos são desde iniciantes, até profissionais experientes. Isso privilegia a troca de experiências e o aprendizado constante da equipe.

Existem no projeto mais de 300 personagens desenvolvidos e pelo menos cinco ambientes prontos onde ocorrerá o jogo. As animações de personagens já estão em sua fase de finalização e exportação, bem como a interface de jogo e HUD já tem protótipo definido, bem como arte finalizada.

Durante o ano de 2015 já foram proferidas três palestras, onde são convidados todos os integrantes do grupo a apresentar o que está sendo desenvolvido. Durante o período do projeto foram publicados cinco artigos em periódicos, um capítulo de livro, oito artigos completos em congressos e quatro resumos. Cabe salientar que todo o grupo participa da escrita de artigos de forma colaborativa, fazendo uso de recursos tecnológicos e ambientes online, onde os participantes mais antigos adotam os mais novos, fomentando e disseminando a pesquisa científica.

Na divisão das atividades, busca-se fazer com que os novos membros da equipe integrem-se com os mais experientes e, com isso, possam aprender nas mais diversas áreas do projeto. A interação social do grupo possibilita grande aprendizado e incentiva a pesquisa e descoberta de novos saberes.

\section{CONSIDERAÇÕES FINAIS}

O Modelo conceitual de Aprendizagem Baseada em Projetos aproxima os conceitos e a prática da aprendizagem colaborativa, onde se embasou a estruturação de um trabalho extraclasse que vem se desenvolvendo ao longo dos últimos dois anos, como meio instrucional.
Para as atividades de aprendizagem colaborativa desenvolvidas, foi definido um conjunto de diretrizes baseadas na metodologia de Projeto E (PROJETOE, 2015). Com isso, foi possível oferecer um ensino de aprendizagem amigável e engajado por parte dos alunos, visando motivar seus integrantes, oferecendo condições de envolvimento, investigação, possibilitando uma maior colaboração entre os pares, proporcionando discussões e reflexões sobre o trabalho desenvolvido, e com isto uma melhora nas ações de planejamentos do projeto, gerando um melhor resultado.

De modo geral é possível citar como benefícios aos participantes, a aprendizagem de ferramentas para gerenciamento de projetos (MSPROJECT, 2015), gerenciamento de defeitos (MANTIS, 2015), modelagem do banco de dados entre outras. Além disso, os colaboradores exercem a prática em desenvolvimento de software com diversas linguagens de programação e utilizam ferramentas para versionamento e desenvolvimento em grupo como Git (GIT, 2015).

A experiência aqui descrita pode se constituir como exemplo para os instrutores na criação de projetos de aprendizagem baseadas em projeto nas mais diversas áreas e na criação dos objetos educacionais. Além disso, oferece uma solução para a coordenação das interações colaborativas do grupo durante a execução de projetos, o que representa uma orientação importante para a aprendizagem colaborativa. Atualmente, o modelo aqui proposto está sendo utilizado de forma experimental, porem já pode ser notado diversos benefícios oriundo da sua utilização.

É importante ressaltar que este trabalho não tem a pretensão de apresentar resultados sobre o uso do Health Simulator, e sim servir para orientar o Modelo conceitual de Aprendizagem Baseada em Projetos no desenvolvimento de simuladores educacionais para área da saúde. 


\section{REFERÊNCIAS}

Barros, P. R. M.; Erh, E.; Bez, M. R.; Cazella, S. C.; Flores, C. D. (2011). Framework para Re-engenharia do Ambiente AMPLIA. In: Anais do V Workshop-Escola de Sistemas de Agentes. v. 2. p. 97-108

Barros, P. R.; Cazela, S. C.; Bez, M. R.; Flores, C. D.; Dahmer, A.; Mossmann, J. B.; Fonseca, J. M.; Maroni, V. (2012) Um Simulador de Casos Clínicos Complexos no Processo de Aprendizagem em Saúde. RENOTE. Revista Novas Tecnologias na Educação, v. 12, p. 1-11.

Bez, M. R. (2013) Construção de um Modelo para o Uso de Simuladores na Implementação de Métodos Ativos de Aprendizagem das Escolas de Medicina. Porto Alegre, 2013. 314 f. Tese (Doutorado PGIE/CINTED- UFRGS, Porto Alegre.

BIE (Buck Institute for Education). (2008) Aprendizagem Baseada em Projetos: guia para professores de ensino fundamental e médio. Porto Alegre: Artmed,

Bradley, P. (2006) The history of simulation in medical education and possible future directions. Medical Education, v. 40, n. 3, p.254-262.

Delors, J. (2006) Educação - Um Tesouro a Descobrir. 10a Edição, Editora Cortez; Brasília - DF; MEC; UNESCO.

Flores, C. D.; Bez, M. R.; Bruno, R. (2011). O Uso de Simuladores de Ensino de medicina. In: Anais ESUD 2011, 2011.v. 1. p. 1-10.

Flores, C. D.; Bez, M. R.; Respício, A.; Fonseca, J. M. (2012) Training Clinical Decision-Making through Simulation. In: Lecture Notes in Business Information Processing. 1ed.Londres: Springer Berlin, 2012, v. 121, p. 59-73.
Flores, C.; Seixas, L; Gluz, J.; Vicari, R. (2005) A Model of Pedagogical Negotiation. In: Carlos Bento, C., Cardoso, A., Dias, G. (eds.) Multi-Agent Systems: Theory and Applications Workshop. 12th EPIA 2005. LNCS 3808, pp. 488-499.

Garret, J.J. (2003) The Elements of User Experience: User Center Design for The Web. New Riders.

GIT. Disponível em: <https://git-scm.com/ >. Acesso em: 10/08/2015.

HIGGS, J., Jones $M$, Loftus S, Christensen, N. (2008).Clinical Reasoning in the Health Professions- 30 Ed.2000:223-234

JSON Introducing JSON. Disponível em: <http://json.org/>. Acesso em: 20/10/2014.

Lacerda, G. S.; WILDT, D. F.; RIBEIRO, V. G. (2004) Uma Introdução às Metodologias Ágeis de Software.

Liker, J. K. (2005) O modelo Toyota: 14 princípios de gestão do maior fabricante do mundo. Porto Alegre: Bookman. p. 316.

Lima, A. Aymone, J., L. F. (2015). Práticas Ágeis Aplicadas ao Desenvolvimento de Jogos Digitais. Novo Hamburgo: Gamepad.

Mafra, B. S. (2014) Uma experiência de aprendizagem significativa de competências de comunicação e expressão em um curso de engenharia: produção escrita de manuais técnicos a partir da interação entre pares. Seminário Internacional de Educação Superior. Mantis. Disponível Em: < Https://Www.Mantisbt.Org/ $>$. Acesso em: 10/08/2015.

Markham, T.; Larmer, J.; Ravitz, J. (2008) In: Buck Institute For Education; . Aprendizagem Baseada em Projetos - guia para professores de ensino fundamental e médio- 2a ed., Porto Alegre: Artmed. 
Maroni, V.; Flores, C. D.; Cazella, S. C.; Bez, M. R.; Dahmer, A. (2013) Development and Evaluation of an Intelligent Pedagogical Agent for the SimDeCS Software. Procedia Technology, v. 9, p. 1217-1226.

M SPROJECT D isponível e m: $<$ https://products.office.com/en-us/project/projectand-portfolio-management-software $>$ Acesso em: 10/08/2015.

Masson, T. J.; Miranda, L. F.; Munhoz Jr, A. H.; Castanheira, A. M. P. (2012) Metodologia de Ensino: Aprendizagem Baseada em Projetos (PBL). In: XL Congresso Brasileiro de Educação em Engenharia, COBENGE, Belém-PA.

Meurer, H.; Szabluk, D. (2010) Projeto E: aspectos metodológicos para o desenvolvimento de projetos dígito-virtuais. Revista da Associação Brasileira de Ergonomia- ISSN 1519-7859. v.5, n.2. Disponível em < http://www.abergo.org.br/revista/index.php/ae/arti cle/view/85>. Acesso em: 20/10/2014.

Microsoft. (2014) Um Guia para a concepção e construção de RESTful Web Services. Disponível em: <ht t p : //m sd n. microsoft.com/en us/library/dd203052.aspx>. Acesso em: 20/10/2014.

Nobre, J. C. S.; Loubach, D. S.; Cunha, A. M.; Dias, L. A. V. (2006) Aprendizagem Baeseada em Projeto (Project-Based Learning PBL) Aplicada a Software Embarcado e de Tempo Real. XVII Simpósio Brasileiro de Informática na Educação.

Novak, J. (2010) Desenvolvimento de Games. 2 ed. SP: Cengage Learning. p. 443.

Orton, E.; Mulhausen, P. (2008) E-learning virtual patients for geratric education. Gerontology \& Geriatrics Education, v. 28, n. 3, p.73-88.

Papinczack, T. (2009) Are deep strategic learners better suited to PBL? A preliminary study. Advances in
Health Sciences Education, v.14. p 337-.353.

Perl, J. (1998) Probabilistic Reasoning in Intelligent Systems: Networks of Plausible Inference. vol.1, 2 ed. p.552, Morgan Kaufmann, San Mateo, CA.

Preece, J.; Rogers, Y.; Sharp, H. (2005) Design de Interação: Além da interação homem-computador. Porto Alegre: Bookman.

Projeto E. (2015) Projeto E - Metodologia Projetual como Modelo de Aprendizagem Baseada em Projetos - Disponível em: http://projetoe.com/aprendizagembaseada-em-projetos/\#sthash.vm5LST1K.dpbs.

Scott, E; Soria, A; Campo, M. R.; Rodriguez, G. H. (2014) Are learning styles useful indicators to discover how students use Scrum for the first time? Computers in Human Behavior, Buenos Aires: v. 36, p. 56-64.

Sebastiani, R. L.; Bez, M. R.; Bruno, R.; Flores, C. D. (2014) Validação do Simulador de Paciente Virtual SIACC. Espaço para a Saúde, v. 15, p. 665-675.

Sebastiani, R. L.; Flores, C. D.; Bez, M. R. (2010). Uso de Imagem para o Ensino em Medicina. In: Anais V LACLO - Congresso Latino- Americano de Objetos de Aprendizagem. v. 1. p. 1-10.

Sebastiani, R. L.; Zago, M. K.; Montovani, A.; Bez, M. R.; Bruno, R.; Dahmer, A.; Flores, C. D. (2012) Ferramenta de Autoria para Construção de Casos Clínicos Interativos para Educação Médica.. In: Anais do XII Workshop de Informática Médica. Porto Alegre: SBC. v. 1. p. 1-10.

Sommerville, I. (2011) Arquitetura orientada a serviços. Engenharia de Software. 9th ed., p.355-368, 2011. São Paulo: Person Prentice Hall.

Sun, L., Zeng, Y., Xiang, Y. (2010) An influence diagram approach for multiagent time-critical dynamic decision modeling. In: Zhang, B., Orgun, M. A. (eds.) 


\section{Ingenierías:H :}

PRICAI 2010. LNCS, v. 6230, pp. 674-680. Springer, Heidelberg.

Teles, V. M. (2006) Extreme Programming: Aprenda como encantar seus usuários desenvolvendo software com agilidade e alta qualidade. São Paulo: Novatec. p. 315.

Wormack, J. P. (2004) A Mentalidade Enxuta nas Empresas: Elimine o Desperdício e Crie Riqueza. Rio de Janeiro: Elsevier. p. 408.

Zeng, Y., Xiang, Y. (2010) Time-critical decision making in interactive dynamic influence diagram. In: IEEE/WIC/ACM International Conference on Intelligent Agent Technology, IAT 2010. Toronto.

Ziv, A.; Ben-David, S.; Ziv, M. (2005) Simulation Based Medical Education: an opportunity to learn from errors. Medical Teacher, v. 27, n. 3, p.193-199. 